\title{
论 文物理、力学和天文中的手征性专题
}

\section{湍流场中涡线单元的结构分析}

\author{
王利坡 \\ 上海交通大学密西根学院, 上海 200240 \\ *联系人, E-mail: Lipo.wang@sjtu.edu.cn \\ 收稿日期: 2019-03-21; 接受日期: 2019-07-08; 网络出版日期: 2019-10-22 \\ 国家自然科学基金(编号: 11172175)资助项目
}

\begin{abstract}
摘要 与描述湍流速度场的流线管单元 [Wang L. On properties of fluid turbulence along streamlines. J Fluid Mech, 2010, 648: 183-203]的思想类似, 为了描述湍流涡矢量场的结构, 我们提出了一种新的结构分析方法, 即涡线单元, 可以用单元尺度和极值点涡量差值来表征. 涡线单元与常用的涡管结构的不同可以概括为: 涡线单元结构可以定 量描述, 并且是全空间填充的. 在理论上这两个条件是保证我们可以将浴线单元的结构分析与全流场统计规律联 系起来的必要前提, 帮助理解和重构湍流涡量场. 根据直接数值模拟数据, 我们研究了涡线单元的统计与结构特 性, 包括尺度与手性等. 根据涡线单元结构可以定量表征精细的条件统计, 例如发现涡相关的动力学参数(例如拟 涡能耗散率和涡拉伸率)强烈依赖于基于涡线单元结构的条件统计. 这样一种结构分析方法为深入研究湍流场提 供了新的手段.
\end{abstract}

关键词 涡矢量, 涡线单元, 手性, 湍流场结构

PACS: 47.27.-i, 47.27.Eq, 47.27.Nz, 47.40.Ki, 47.85.Gj

\section{1 概述}

在湍流状态下, 尤其是高雷诺数的情况, 对流作用 和边界条件使得湍流系统具有很强的非线性和非局部 性. 再加上复杂的作用源项使得湍流问题本身无法仅 仅根据控制方程而获得自洽的信息. 所以只从数学角 度去认识湍流在目前阶段存在着一些无法克服的困 难, 我们需要从不同的角度寻求突破. 例如经典的Kolmogorov理论并不是从控制方程本身推导出来的 ${ }^{[1]}$. 一 种可能的方向是通过分析流场的几何结构来认识这一 复杂现象 ${ }^{[2]}$.
浴矢量 $\boldsymbol{\omega}$ 是描述流体力学的一个重要物理量, 在 湍流研究中有着重要作用, 其定义如下: $\boldsymbol{\omega}=\nabla \times \mathbf{u}$. 大 量的文献中讨论了 $\boldsymbol{\omega}$ 的数学和拓扑特征 ${ }^{[3,4]}$. 由于不同 尺度下流体运动的耦合，压缩区和拉伸区相互作用使 得浴量局部集中. 高浴量区域的空间分布与湍流间歇 性密切相关. 通常平均来看, 浴更容易被拉伸而不是 被压缩 ${ }^{[3,4]}$. 从动力学上来说，浴旋拉伸导致在不同尺 度上都有速度脉动 ${ }^{[5,6]}$. 这一拉伸机制对于理解三维湍 流中一些最本质的特征十分重要 ${ }^{[3]}$.

由于稳定性原因，具有明显浴量的区域更倾向于 聚集组织成管状 ${ }^{[7,8]}$, 被称为涡管. Moffatt等人 ${ }^{[9]}$ 将浴

引用格式: 王利坡. 湍流场中浴线单元的结构分析. 中国科学: 物理学 力学 天文学, 2020, 50: 040003

Wang L P. Analysis of the vorticity tube segment in fluid turbulence (in Chinese). Sci Sin-Phys Mech Astron, 2020, 50: 040003, doi: 10.1360/SSPMA2019-0086 
管描述成湍流场的筋腱，以强调在塑造流场结构方面 的特殊性. 通常认为浴管的长度和半径分别与泰勒尺 度和耗散尺度在同一数量级. 细致的拓扑研究显示, 浴旋线的平均曲率半径约等于泰勒尺度 ${ }^{[10]}$. 沿着浴旋 线，不同的特征参数具有不同关联尺度. 更高雷诺数 下的直接数值模拟结果表明，涡结构的特征尺度仍然 很难有定论 ${ }^{[1]}$. Ruetsch和Maxey ${ }^{[11]}$ 表明能量耗散与浴 管有关.一般耗散的区域倾向于围绕浴管，但强烈耗 散区域倾向于存在于两个或更多个相邻的浴管之间. 通常, 强耗散区域与强浴旋区域并不重叠. 通过模拟数 据可视化结果可以清楚地看到大尺度螺旋结构和涡管 相互作用的各种演化模式 ${ }^{[12]}$.

虽然进行了广泛的研究, 但浴旋结构的识别仍然 存在争议 ${ }^{[13,14]}$. 例如，用一些预设的阈值来定义浴管 会引入非客观的任意性. Haller ${ }^{[13]}$ 表明浴旋的定义在 一般坐标变换下应该是不变的，在此基础上新的涡旋 定义应该与框架无关. 浴管基本对应流场中的高湍流 强度区域，尽管表征不同，但仅占整个流场的非常小 的体积(基本上小于 $10 \%$ ). 因此仅仅通过浴管无法合 适地表示湍流场. 换言之, 浴管的性质可能并不总是 与全湍流场的性质密切关联. 为了合理的修正，首先 必须客观地定义一种全空间填充几何结构. 然而, 目 前为止并没有成熟的方法可以使用.

关于流场结构分析，近年来已经开发了一些新方 法来关注湍流的拓扑特征. 例如对于流场中给定的某 种标量, Wang和Peters ${ }^{[15]}$ 提出了梯度线元(Dissipation Element, DE)结构将标量场合理划分. 从任何一空间 点出发，该标量的梯度轨迹沿着正方向会到达一个局 部极大值，沿负方向会到达局部极小值.一个梯度线 元是梯度轨迹具有同一对局部最小和最大值点的所有 空间点的集合. 由于全流场是由空间点组成, 所有梯度 线元必须空间填充. 我们可以从这些相对简单的分解 结构更好地理解复杂的全流场. 具体而言, 如果分解 结构可以通过参数组 $(p 1, p 2, \ldots)$ 来刻画，对于某种性 质 $X$, 分解单元中的 $X(p 1, p 2, \ldots)$ 相对容易建模. 如果 知道参数组 $(p 1, p 2, \ldots)$ 的联合概率密度函数 (PDF) $P(p 1, p 2, \ldots)$, 那么根据

$$
\langle X\rangle=\int \ldots \int X(p 1, p 2, \ldots) P(p 1, p 2, \ldots) \mathrm{d} p 1 \mathrm{~d} p 2 \ldots,
$$

可以预测全场的统计平均 $\langle X\rangle$. 注意到公式(1)中的积
分成立条件对应着结构的空间填充.

原则上，此方法适用于不同的标量 ${ }^{[16]}$. 对于矢量 场, 梯度线元结构无法直接定义, 需要发展新的诊断 方法.

一个有趣的尝试是矢量管单元结构 ${ }^{[17]}$. 对于速度 场，可以使用流线管单元来研究全场. 矢量管单元同 样是空间填充的. 对于具体的统计结果, 根据矢量管 单元的体积做权重平均等价于经过空间均匀分布的矢 量线单元的样本平均 ${ }^{[17]}$. 以速度矢量为例, 根据特征 参数, 流线单元可以定义为正或负. 一个有启发性的 结论是, 由于正负单元之间的不对称性, 速度导数的 偏斜度是一个自然的动态演化结果. 与梯度线元结构 类似, 流线单元方法通用于不同的矢量场分析.

浴量和速度矢量在湍流运动学和动力学中都是至 关重要的. 本文将运用类似流线单元的思想来研究湍 流浴量场, 尤其是研究湍流场的非局部性质和条件 统计.

\section{2 几何定义}

在矢量场中, 与矢量局部相切的矢量线, 反映了给 定矢量场内在拓扑结构. 对于速度和浴量情况, 矢量线 分别命名为流线和浴线. 矢量线和标量变量中的梯度 轨迹一样具有清晰的物理含义.

考虑不可压缩情况的浴量方程

$\frac{\partial \boldsymbol{\omega}}{\partial t}+\mathbf{u} \cdot \nabla \boldsymbol{\omega}=v \nabla \cdot \nabla \boldsymbol{\omega}+\boldsymbol{\omega} \cdot \nabla \mathbf{u}$,

其中 $v$ 是运动黏度, $\mathbf{u}$ 是速度矢量. 将 $\boldsymbol{\omega}$ 和 $\mathbf{u}$ 的单位方向 矢量分别表示为 $\mathbf{t}$ 和 $\mathbf{t}_{\mathbf{u}}$. 这样浴量和速度矢量分别表示 为 $\omega \mathbf{t}$ 和 $u \mathbf{t}_{\mathbf{u}}$. 方程(2)两边同时乘上 $\mathbf{t}$ 得到

$\frac{\partial \omega}{\partial t}+u \frac{\partial \omega}{\partial s_{u}}=v \mathbf{t} \cdot \nabla \cdot \nabla \boldsymbol{\omega}+\omega\left(\mathbf{t} \cdot \frac{\partial \mathbf{u}}{\partial s}\right)$,

其中 $s$ 和 $s_{u}$ 分别是沿着浴线和流线的曲线坐标. 将方程 (3)乘上 $\omega$, 我们得到拟浴 $\omega^{2} / 2$ 的方程如下:

$\frac{\partial\left(\omega^{2} / 2\right)}{\partial t}+u \frac{\partial\left(\omega^{2} / 2\right)}{\partial s_{u}}=v \omega \mathbf{t} \cdot \nabla \cdot \nabla \boldsymbol{\omega}+\mathcal{P}$,

其中

$\mathcal{P}=\omega^{2} \mathbf{t} \cdot \frac{\partial \mathbf{u}}{\partial s}$

为拟浴项, 是理解浴旋动力学的关键参数, 之后将讨论 
其性质. 公式(3)和(4)沿浴线的变换形式在于强调流场 的几何意义.

与标量的梯度轨迹不同，浴线或一般的矢量线大 多是未闭合且无限长的. 这里采用类似于流线单元 ${ }^{[17]}$ 的方法来分割浴线. 如图1所示，在浴量场中，任何空 间点沿其浴度线，可以达到涡量 $\omega$ 的局部最大值和最 小值. 由两个相邻极值点(一个极大值和一个极小值) 界定的浴线部分被定义为相对于给定点的浴线单元. 划分浴线单元可以灵活地选择判据，例如浴量的极值 或其他物理量的极值. 通常对于一般矢量场来说, 关 联最为密切的参数是其矢量的模值，因此在本文中我 们根据 $\omega$ 的极值来划分浴线单元. 来自相邻浴线的极 值点集构成极值曲面．沿浴量矢量方向，起始点 $\omega_{s}$ 处 的浴量幅值在终点处单调变化为 $\omega_{e}$. 两个极值点之间 的弧长 $l$ 和 $\Delta \omega=\omega_{e}-\omega_{s}$ 用于定量表征浴线单元. 根据 $\Delta \omega$ 的符号，如果 $\Delta \omega$ 分别为正和为负，则该浴线单元定义 为正单元或负单元.

\section{3 结果与分析}

\section{1 直接数值模拟}

我们的统计分析基于均匀剪切流动的直接数值模 拟(DNS)数据. 如图 2 所示, 在 $2 \pi$ 立方体内的均匀剪切 湍流通过一平均速度梯度 $S=\mathrm{d} u_{1} / \mathrm{d} x_{2}$ 作为湍流源项，结 合运动黏度 $v$ 来控制湍流强度.

参考坐标系选择随平均流运动，所以控制方程中 作为因变量的速度沿三个方向 $x_{i}(i=1,2,3)$ 满足周期 性边界条件，从而可以采用傅里叶谱方法用于空间导 数求解. 压力通过泊松方程在谱空间求解. 时间推进 采用三阶Runge-Kutta算法，时间步长 $\Delta t$ 遵循如下的 Courant条件:

$(\Delta t)^{-1}=2\left(\frac{\left|u_{1}\right|}{\Delta x_{1}}+\frac{\left|u_{2}\right|}{\Delta x_{2}}+\frac{\left|u_{3}\right|}{\Delta x_{3}}\right)_{\max }$.

我们采用了Feiereisen等人 ${ }^{[18]}$ 的算法以满足连续 性方程. 为了确保分辨率足够精细以提取基本的几何 特征, 网格尺寸需要小于Kolmogorov尺度, 即 $\Delta x$ / $\eta<1^{[15,17]}$.

移动框架的问题在于, 随着时间的推移, 物理空间 中的计算域变得越来越偏斜. 因此, 需要重构变形框架 以避免过度扭曲. 如图3所示，原始笛卡尔网格由状态

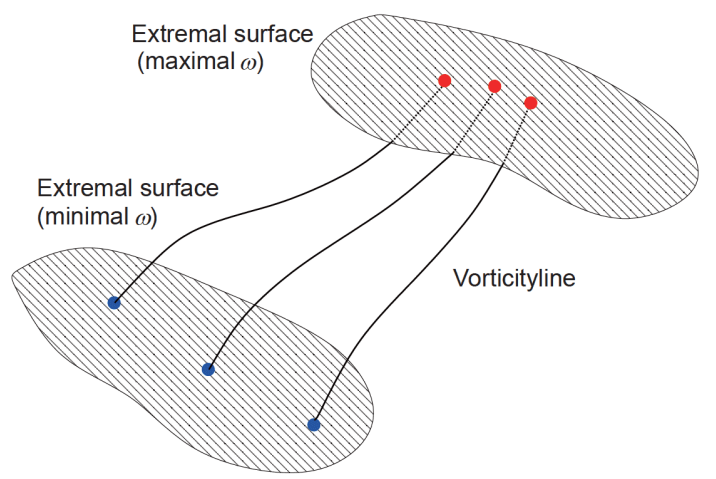

图 1 (网络版彩图)浴线单元结构示意

Figure 1 (Color online) Schematic of vorticityline segments.

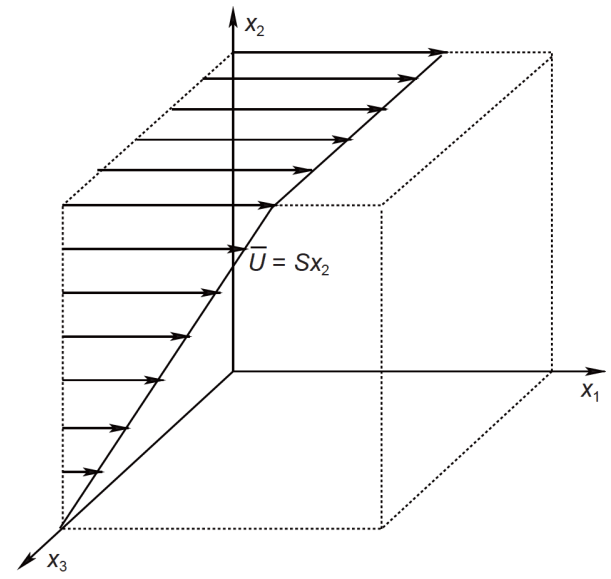

图 2 在 $2 \pi$ 立方体内的均匀剪切流动构型

Figure 2 Flow configuration of homogeneous shear turbulence in a $2 \pi$ cubic domain.

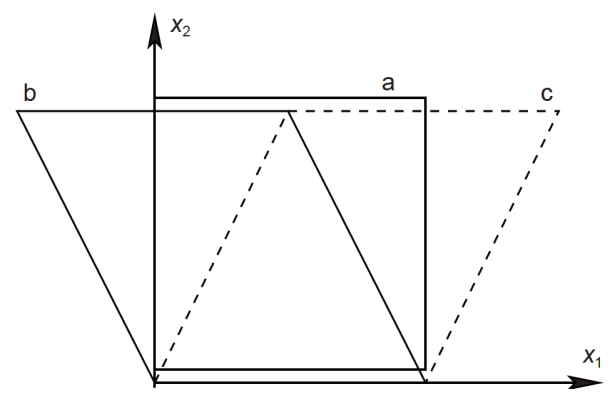

图 3 网格重构示意图

Figure 3 Frame regriding.

(a)连续变形直到状态 (c), 然后重构网格为状态(b). 是 否需要重构需要在计算中实时判断.

原则上, 从初始条件来看, 均匀剪切湍流中的积分 尺度将随时间呈指数增长. 在这种意义上, 由于计算域 
有限, 不存在精确的均匀剪切湍流模拟. 数值上, 如果 模拟时间足够长, 最大尺度将达到域边界, 湍流能量将 部分释放以收缩最大尺度，然后再次增加，依此类推. 换句话说, 浴旋大小随时间而波动. 在数据分析中, 仅 考虑波动变量. 数值试验表明, 边界干扰不会对统计结 果产生明显影响. 例如, 参数 $S k / \varepsilon$ 代表能量产生和耗散 的平衡，其中 $k$ 和 $\varepsilon$ 分别代表了平均湍流动能和能量耗 散. 数值结果表明平均上, 参数 $S k / \varepsilon$ 约为 $4-5$, 与理论预 测结果吻合较好. 在计算网格为 $2048^{3}$ 的条件下，流体 黏性系数 $v=0.0009$ 及 $S=1.5$ 的给定参数下，基于泰勒尺 度 $\lambda$ 的雷诺数 $R e_{\lambda}=\sqrt{2 k} \lambda / v$ 为 295 .

\section{2 拓扑特征与手性}

首先对于均匀分布的网格点的DNS情况，各个网 格具有相同的权重. 为了保证统计的无偏差, 所以网 格点的浴线段需要无遗漏地采样. 另外识别浴线段的 关键因素是极值定义. 与速度矢量情况类似，数值上 浴量的极值通过检查 $\mathbf{t} \cdot \nabla \boldsymbol{\omega} \cdot \mathbf{t}$ 的符号来确定 ${ }^{[17]}$. 这一 准则被证明优于其他标准. 通过矢量线的结构描述算 法 $^{[15]}$, 经过空间任一网格点的浴线单元可以有效获得.

在流体动力学中, 速度与浴量相互作用. 如图4所 示, 在任何一网格点, 可以定义相应的流线单元(SS)和 浴线单元(VS), SS 和VS的弧长分别由 $l_{u}$ 和 $l$ 表示. 图5 显 示了 $l_{u}$ 和 $l$ 的联合 $\mathrm{PDF}$ 及条件平均 $l_{u} \mid l$ 和 $l \mid l_{u}$ (分别为 $l_{u}$ 关于 $l$ 的条件平均及 $l$ 关于 $l_{u}$ 的条件平均). 总体而言, $l_{u}$ 和泣间没有明显的相关性, 并且条件平均值近似恒定.

浴量描述了速度的浴旋，但无法表述局部旋转的 另外一个重要属性，速度场的手性. 如图6所示，在局 部浴量场相同的情况下，(a)和(b)对应着不同的手性, 或者说不同的手性强弱. 关于手性强度的表征有着很 多不同的描述方法 ${ }^{[19]}$. 根据直观的几何特性，我们可 以用局部速度与浴矢量的夹角 $\theta$ 来刻画. 可以明显地 看到，在图6(a)和(b)中由于 $\cos \theta$ 符号的不同，导致了速 度的不同手性.

图7表示空间网格点处 $\cos \theta$ 的统计平均随经过该 点的浴线单元尺度了 $l$ 与该点的浴矢量模值 $|\omega|$ 的变化. $l$ 较小时, $|\omega|$ 平均较大; l较大时, $|\omega|$ 平均较小. 在 $|\omega|$ 和 $l$ 为中间值时 $\cos \theta$ 趋近于 1 , 而在其他区域, $\cos \theta$ 较小. 这 一结果清晰地表明，在统计上手性的强弱和浴线单元 的几何特征有紧密联系. 更详细的挖掘需要深入研究.

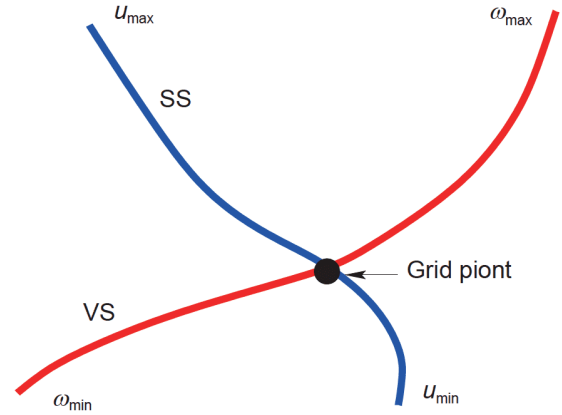

图 4 (网络版彩图)经过任何一空间点, 同时可以定义相应 的浴线单元(VS)和流线单元(SS). 这两种结构的空间关联反 应了湍流场的性质

Figure 4 (Color online) A vortexline segment (VS) connects with a streamline segment (SS) at a joint grid point. The correlation between VS and SS inherits the turbulence physics.

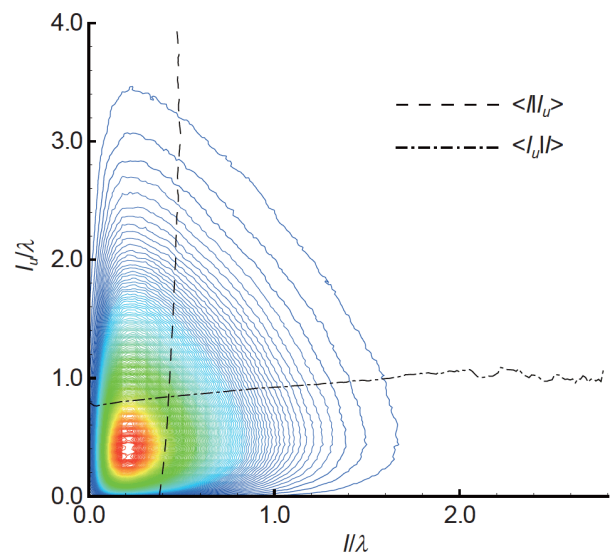

图 5 (网络版彩图) $l_{u}$ 和 $l$ 的联合概率密度函数 (PDF)与条件 平均 $l_{u} \mid l$ 和 $l \mid l_{u}$

Figure 5 (Color online) Joint PDF of $l_{u}$ and $l$, together with the conditional means.
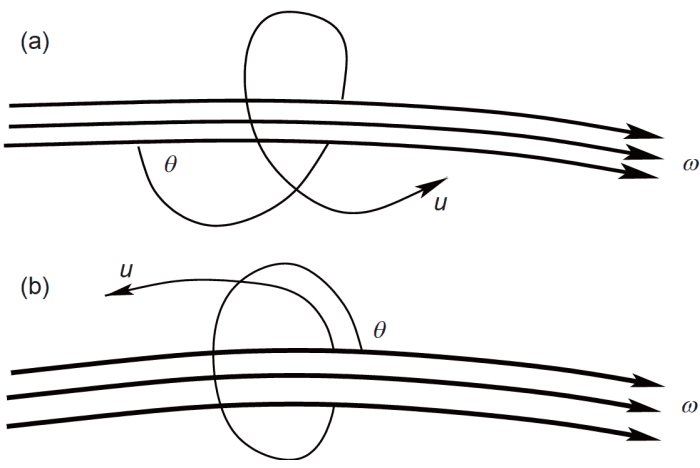

图 6 (a)与(b)对应着相同的局部浴矢量，但具有不同的手 性. 该特性可以用局部速度与涡矢量的夹角 $\theta$ 来刻画

Figure 6 Case (a) and (b) have different chirality, but share the same local vorticity vector, which can be characterized by the $\theta$ quantity. 


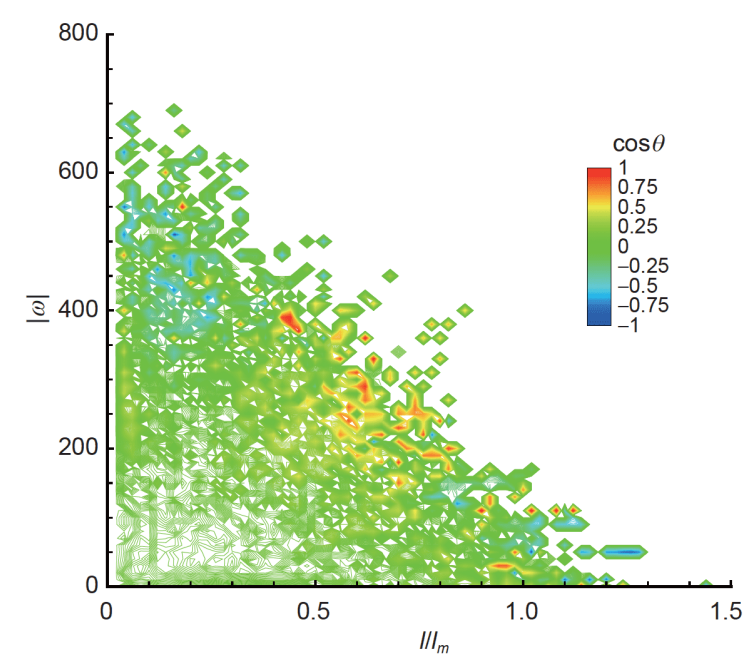

图 7 (网络版彩图) $\cos \theta$ 在浴线单元结构中的条件平均 Figure 7 (Color online) Conditional average of $\cos \theta$ in the framework of the vorticity line segment structure.

考虑特征参数, 即弧长 $l$ 和两个极值之间的 $\omega$ 差 $\Delta \omega=\omega_{e}-\omega_{s}$. 对正涡线单元 $\Delta \omega$ 是正, 反之亦然. $l$ 和 $\Delta \omega$ 的联合概率密度函数如图8(a)所示. 就正 $\Delta \omega$ 和负 $\Delta \omega$ 部 分而言，图8(a)表现为总体对称. 对于具有特征参数 $l_{u}$ 和 $\Delta u$ 的流线单元的结果如图 8(b)所示. 对于正和负流 线单元，速度分别沿速度矢量方向单调增加和减少. 因此，当随时间演变时，具有增加速度的正流线单元 倾向于被拉长，而负流线单元则被压缩。这种运动效 应解释了图8(b)的强烈不对称性和负的速度导数偏斜 度 ${ }^{[17]}$. 对于浴量情况，这种不对称的拉伸和压缩机制 不存在，因此图8(a)和(b)中的联合概率密度函数也表 现不同。

弧长 $l$ 是描述几何和流动物理的关键参数. 在研究 耗散元的尺度的工作中已经有了一些开创性的成果. Wang和Peters ${ }^{[16]}$ 提出了以下关于归一化尺度 $\tilde{l}=l / l_{m}$ 的概率密度函数的模型方程 $\left(l\right.$ 用自己的平均值 $l_{m}$ 归 一化):

$$
\begin{aligned}
& \frac{\partial \widetilde{P}(\tilde{l}, t)}{\partial t}+\frac{\partial}{\partial \widetilde{l}}(\tilde{v}(\tilde{l}) P(\tilde{l}, t)) \\
& \left.=\Lambda \int_{0}^{\infty} 2 P(\widetilde{l}+\widetilde{z}, t) \mathrm{d} \tilde{z}-\widetilde{l} \widetilde{P}(\widetilde{l}, t)\right] \\
& \quad+\left.8 \frac{\partial \widetilde{P}(\widetilde{l}, t)}{\partial \widetilde{l}}\right|_{\tilde{l}=0}\left[\int_{0}^{\tilde{l}} \frac{\widetilde{z}}{\widetilde{l}} P(\widetilde{l}-\widetilde{z}, t) P(\widetilde{z}, t) \mathrm{d} \tilde{z}-P(\widetilde{l}, t)\right] .
\end{aligned}
$$
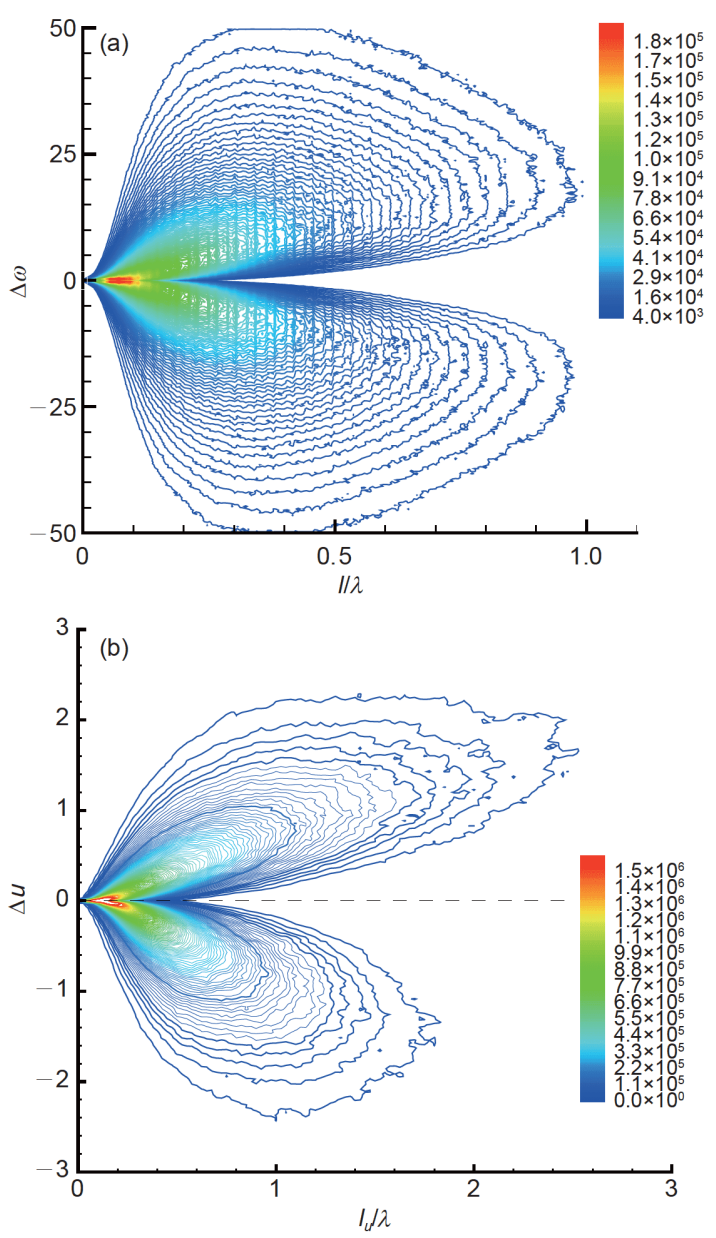

图 8 (网络版彩图)结构特征值的联合PDF，(a) 涡线单元; (b) 流线单元. 两种PDF表现出对称性不同反应了两种矢量 场的统计特性的差异

Figure 8 (Color online) Joint PDF of the characteristic parameters for (a) voriticityline segments; (b) streamline segments. The symmetry difference indicates the difference between these two vector fields.

在上面的等式中, $\widetilde{v}(\widetilde{l})$ 是描述极值点相对于彼此 的运动的漂移速度， $\Lambda$ 是可以根据概率密度函数的归 一化条件确定的特征值. 在物理上， $l$ 的演变是不同机 制共同作用的结果. 首先, 黏性扩散产生的平滑机制可 以消除极值点以平滑流动轮廓; 其次, 湍流运动导致的 扰动机制将扰乱流动轮廓以产生新的极值点. 这两种 机制使极值点的数目发生突变从而使 $l ⿺_{2}$ 生突变. 另外 漂移速度 $\tilde{v}(\widetilde{l})$ 则渐进改变单元尺度 $l$. 在统计稳态条件 下，尺度 $l$ 在上述因素的控制下达到动态平衡. 原则上, 该物理过程不仅能够描述耗散元长度的变化，而且通 常也适用于其他尺度演变过程. 达到统计稳态时，方 程(6)中的时间导数项消失, 相应的方程的数值求解已 
在参考文献[15]中讨论过. 图9表示由DNS数据分析全 场浴线单元而得到的 $\tilde{l}$ 概率密度函数. 可以看到, 无论 在线性-线性和对数-线性坐标中, 数值结果与方程(7) 的理论解相比，都有满意的结果. 尤其是在大尺度范 围( $(\tilde{l}>2.5)$, 模型结果与测量结果呈现出指数减少的 规律. 这是由于单元尺度基本满足Poisson随机过程导 致的自然结果 ${ }^{[15]}$.

\section{3 条件统计}

通过将空间划分为相对简单的结构单元，可以进 一步研究以几何特征为基础的条件统计，以便更深入 地理解流动的物理过程.

一个普遍认可的观点是，浴量更容易拉伸而不是

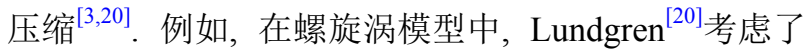
拉伸的浴结构诱导流场并可以合理地预测能谱的一些 性质. 浴量-应变关系在有化学反应的湍流场中也广泛 关注. 参考文献[20]中提到方向角 $\theta=\tan ^{-1} \frac{|\boldsymbol{\omega} \times S \cdot \boldsymbol{\omega}|}{\boldsymbol{\omega} \cdot S \cdot \boldsymbol{\omega}}$ 的 $\mathrm{PDF}$ (其中 $S$ 是应变率张量). 结果表明在 $\cos \theta=1$ 时 PDF 达到最大值, 这个结果与涡旋拉伸的物理理解一致.

考虑沿浴线段的速度投影, 即 $u_{t}=\mathbf{u} \cdot \mathbf{t}$. 两个极值 点的 $u_{t}$ 之差是 $\Delta u_{t}=u_{t e}-u_{t s}$, 沿浴线段的平均应变是 $\Delta u_{t} / l$. 图10(a)显示了根据不同 $\Delta \omega$ 值的所做的关于正涡 线单元 $\Delta u_{t}$ 的条件PDF. 对于负浴线段，结果几乎相同.

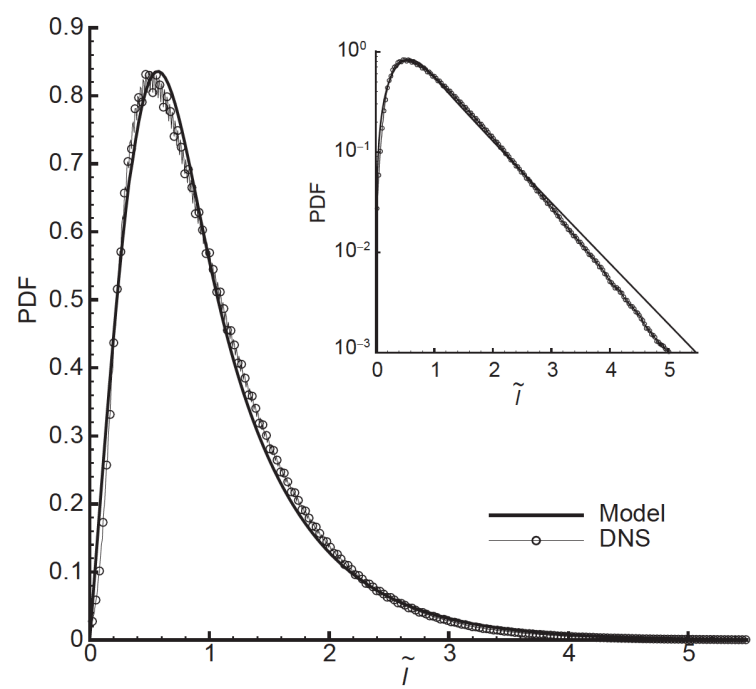

图 9 归一化浴线单元尺度的PDF, 与模型解满意吻合

Figure 9 PDF of the normalized segment arclength, in satisfactory agreement with the model solution.
总的来说，对于小 $\Delta \omega \mid$ 的浴线单元， $P\left(\Delta u_{t}\right)$ 更接近于对 称，而对于大 $|\Delta \omega|$ 值的浴线单元， $P\left(\Delta u_{t}\right)$ 更偏向于为正. 图10(b)显示出了相同样本划分的平均应变率 $\Delta u_{t} / l$ 的 条件PDF.

表1中列出了由图10中计算出的不同样本区间的 $\Delta u_{t}$ 和 $\Delta u_{t} / l$ 的平均值，即 $\overline{\Delta u_{t}}$ 和 $\overline{\Delta u_{t} / l}$. 一个明显的趋势 是, 随着 $|\Delta \omega|$ 的增加, $\overline{\Delta u_{t}}$ 和 $\overline{\Delta u_{t} / l}$ 从负到正单调地增加. 根据这种结果再考虑到之前浴量更容易拉伸的说法, 目前的分析也表明这一结论的合理. 但是, 更精确的结 果是，具有小 $|\Delta \omega|$ 值条件PDF几乎没有偏斜，即浴线单 元既不拉伸也不压缩; 只有那些大 $|\Delta \omega|$ 值的浴线单元 倾向于被拉伸. 拉伸强度强烈取决于 $|\Delta \omega|$ 值, 即浴线单
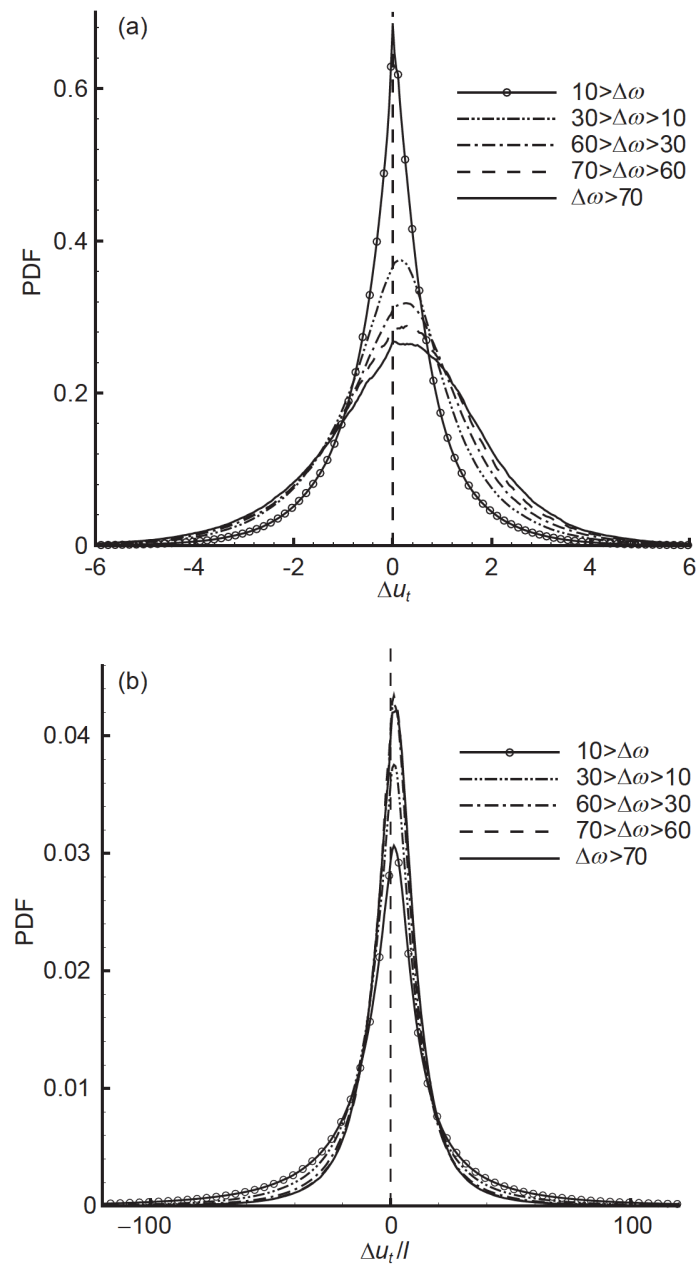

图 10 在不同样本空间得到的关于(a) $\Delta u_{t} ;$, (b) $\Delta u_{t} / l$ 的条件 统计PDF

Figure $10 \quad \mathrm{PDF}$ of (a) $\Delta u_{t}$ and (b) $\Delta u_{t} / l$ from conditional statistics in different categories. 
表 $1 \quad \overline{\Delta u} t_{t}$ 和 $\overline{\Delta u_{t} / l}$ 在不同样本空间的条件平均

Table 1 Conditional means $\overline{\Delta u_{t}}$ and $\overline{\Delta u_{t} / l}$ for different categories

\begin{tabular}{cccccc}
\hline 统计量 & $10>\Delta \omega$ & $30>\Delta \omega>10$ & $60>\Delta \omega>30$ & $70>\Delta \omega>60$ & $\Delta \omega>70$ \\
\hline$\overline{\Delta u_{t}}$ & $-1.381 \times 10^{-2}$ & $1.287 \times 10^{-2}$ & $6.725 \times 10^{-2}$ & 0.115 & $0.138^{2}$ \\
$\overline{\Delta u_{t} / l}$ & $-3.405 \times 10^{-2}$ & $-3.611 \times 10^{-2}$ & $2.264 \times 10^{-2}$ & $3.928 \times 10^{-2}$ & $4.670 \times 10^{-2}$ \\
\hline
\end{tabular}

元的两个端点处浴度值之差. 从流动物理上理解, 平均 应变 $\Delta u_{t} /$ 源自压力变化. 对于具有大 $|\Delta \omega|$ 值的浴线段, 如果假设一个恒定的背景压力，从浴线单元起点到终 点，离心力将产生很大的压力差; 因此在这种情况下, 平均应变会很显著. 这里要强调的是 $|\Delta \omega|$ 而不是 $\omega, \omega$ 本 身不会导致压力变化和局部应变.

在拟浴方程(4)中，由方程(5)定义的拟浴产生项 $\mathcal{P}$ 对于保持总能量的平衡至关重要. 形式上, $\mathcal{P}$ 由浴量和 局部应变(速度梯度)之间的相互作用产生. 物理上, $\mathcal{P}$ 必须是正的以平衡能量耗散，这是从二维湍流与三 维湍流之间的最显著的不同之一。通过观察 $\boldsymbol{\omega}$ 与应变 率张量的特征向量之间的角度的概率密度函数, Tsino$\mathrm{ber}^{[3]}$ 提供了对 $\mathcal{P}$ 为正这一现象的解释. 在浴管单元分 析中，方程(5)中定义的拟涡能产生项 $\mathcal{P}$ 可以沿涡线表 达为

$\omega^{2} \mathbf{t} \cdot \frac{\partial \mathbf{u}}{\partial s}=\omega^{2} \mathbf{t} \cdot \frac{\partial(\mathbf{u} \cdot \mathbf{t})}{\partial s}-\omega^{2} \mathbf{u} \frac{\partial \mathbf{t}}{\partial s}$,

等式右边的最后一项是方向波动. 由于空间方向的随 机性，平均来说这一项的净贡献可以忽略不计. 因此, $\mathcal{P}$ 的平均值可以估计为

$$
\begin{aligned}
\overline{\omega^{2} \mathbf{t} \cdot \frac{\partial \mathbf{u}}{\partial s}} & =\overline{\omega^{2} \mathbf{t} \cdot \frac{\partial(\mathbf{u} \cdot \mathbf{t})}{\partial s}}-\overline{\omega^{2} \mathbf{u} \frac{\partial \mathbf{t}}{\partial s}} \sim \overline{\omega^{2} \mathbf{t} \cdot \frac{\partial(\mathbf{u} \cdot \mathbf{t})}{\partial s}} \\
& \sim \overline{\omega^{2} \Delta u_{t} / l} .
\end{aligned}
$$

因为沿着浴线, $\omega$ 的极值可以随机出现, 即浴线单 元可以在任何地方随机切割, 因此合理地认为 $\omega$ 和 $\Delta \omega$ 相互独立．类似的论证已被证实，例如在充分发展的 各向同性的湍流标量和标量耗散率几乎是独立的 ${ }^{[21]}$, 尤其是在更高雷诺数的情况下. 因此, $\Delta u_{t} / l$ 是决定 对 $\mathcal{P}$ 的贡献的主导因素. 正如图10所示, 对于小 $|\Delta \omega|$ 值 的浴线单元, $P\left(\Delta u_{t}\right)$ 的对称性导致了小的 $\overline{\Delta u_{t} / l}$ 值, 因 此导致小的 $\overline{\omega^{2} \Delta u_{t} / l}$ 值. 而对 $\mathcal{P}$ 的正值性的主要贡献来
自于大 $|\Delta \omega|$ 值的浴线单元, 因为相应的 $P\left(\Delta u_{t}\right)$ 在很大程 度上是正偏移的. 总体而言, 净拟浴能源项必须为正. 更普遍地说，以上相同的观点可以总结为下列关系:

$\overline{\omega^{n} \frac{\partial(\mathbf{u} \cdot \mathbf{t})}{\partial s}} \sim \overline{\omega^{n} \Delta u_{t} / l}>0$,

其中 $n$ 可以取任何值. 例如, 对于 $n=1$ 的情况, 可以沿笛 卡尔坐标系框架 $\left|\omega_{i}\right| a$ 中的 $i=1,2,3$ 个方向检查, 其中 $a$ 是局部应变. DNS数据分析表明沿着 $x_{1}, x_{2}$ 和 $x_{3}$ 方向, 结 果分别为 $4.91,3.18$ 和 3.79 .

\section{4 结论}

从几何结构认识湍流是为了更好地定量刻画流 场, 而不是仅仅做定性的阐述性解释. 另外, 全空间填 充这一重要先决条件保证了几何结构的统计特性可以 用来表征全流场. 本文借鉴了用于研究湍流速度场的 流线单元结构, 引入所谓的浴线单元来研究湍流浴 矢量.

浴管单元的特征参数可以用单元弧长和两个相邻 极值之间 $\omega$ 的差值 $\Delta \omega$ 来定义. 通过DNS数据分析, 我们 发现这两个特征参数的联合概率密度函数对称，这与 流线单元的统计结果情况不同. 弧长的概率密度函数 遵循尺度演化模型方程. 在物理机理分析, 单元弧长 演化受到黏性扩散、湍流扰动和极值点漂移等过程的 共同作用.

根据 $\Delta \omega$ 大小做的浴拉伸特性的条件统计是另外 一个有趣的结果，帮助我们重新认识了浴量更易拉伸 而非压缩的普遍观点. 目前的结果表明只有大的 $|\Delta \omega|$ 值的浴线单元趋向于被拉伸, 而那些小 $|\Delta \omega|$ 值的浴线 单元没有明显的拉伸倾向，即拉伸和压缩作用近似相 当. 这一性质与拟浴能源项的正值性密切相关. 


\section{参考文献}

1 Constantin P, Fefferman C. Scaling exponents in fluid turbulence: Some analytic results. Nonlinearity, 1994, 7: 41-57

2 Moffatt H K, Tsinbor A. Topological fluid mechanics. In: Proceedings of the IUTAM Symposium. Cambridge: Cambridge University Press, 1989

3 Tsinober A. An Informal Introduction to Turbulence. Dordrecht: Kluwer, 2001

4 Moffatt H K, Tsinober A. Helicity in laminar and turbulent flow. Annu Rev Fluid Mech, 1992, 24: 281-312

5 Synge J L, Lin C C. On a statistical model of isotropic turbulence. Trans R Soc Canada, 1943, 37: 45-79

6 Aivazis K A, Pullin D I. On velocity structure functions and the spherical vortex model for isotropic turbulence. Phys Fluids, 2001, 13: 20192029

7 Townsend A A. On the fine-scale structure of turbulence. Proc R Soc Lond A, 1951, 208: 534-542

8 She Z S, Jackson E, Orszag S A. Intermittent vortex structures in homogeneous isotropic turbulence. Nature, 1990, 344: 226-228

9 Moffatt H K, Kida S, Ohkitani K. Stretched vortices - the sinews of turbulence; large-Reynolds-number asymptotics. J Fluid Mech, 1994, 259: $241-264$

10 Jiménez J, Wray A A. On the characteristics of vortex filaments in isotropic turbulence. J Fluid Mech, 1998, 373: 255-285

11 Ruetsch G R, Maxey M R. Small-scale features of vorticity and passive scalar fields in homogeneous isotropic turbulence. Phys Fluids A-Fluid Dyn, 1991, 3: 1587-1597

12 Horiuti K, Fujisawa T. The multi-mode stretched spiral vortex in homogeneous isotropic turbulence. J Fluid Mech, 2008, 595: 341-366

13 Haller G. An objective definition of a vortex. J Fluid Mech, 1999, 525: 1-26

14 Kolář V. Vortex identification: New requirements and limitations. Int J Heat Fluid Flow, 2007, 28: 638-652

15 Wang L, Peters N. The length-scale distribution function of the distance between extremal points in passive scalar turbulence. J Fluid Mech, 2006, 554: $457-475$

16 Wang L, Peters N. Length-scale distribution functions and conditional means for various fields in turbulence. J Fluid Mech, 2008, 608: 113-138

17 Wang L. On properties of fluid turbulence along streamlines. J Fluid Mech, 2010, 648: 183-203

18 Feiereisen W J, Reynolds W C, Ferziger J H. Numerical simulation of compressible, homogeneous, turbulent shear flow. Report TF-13. Department of Mechanical Engineering, Stanford University, 1981

19 Petitjean M. Chirality and symmetry measures: A transdisciplinary review. Entropy, 2003, 5: 271-312

20 Lundgren T S. Strained spiral vortex model for turbulent fine structure. Phys Fluids, 1982, 25: 2193-2203

21 Fox R O, Hill J C, Gao F, et al. Stochastic modeling of turbulent reacting flows. In: Proceedings of the 1992 Summer Program. Center for Turbulence Research, Stanford University, 1992 


\title{
Analysis of the vorticity tube segment in fluid turbulence
}

\author{
WANG LiPo* \\ UM-SJTU Joint Institute, Shanghai Jiao Tong University, Shanghai 200240, China
}

\begin{abstract}
To address the geometrical properties of the turbulent velocity vector field, a new concept named streamtube segment has been developed [Wang L. On properties of fluid turbulence along streamlines. J Fluid Mech, 2010, 648: 183-203]. According to the vectorial topology, the entire velocity field can be partitioned into the so-called streamtube segments, which are organized in a non-overlapping and space-filling manner. A similar idea is implemented to study the turbulent vorticity vector field. Differently from the conventional vortex tubes, vorticity tube segments are space-filling and can be characterized by non-arbitrary parameters. Such structure enables a more quantitative, rather than illustrative, description of turbulence. In principle, these two conditions are the linkage to reproduce the entire turbulent field from decomposed geometrical units with relatively simple structures. From analyzing the direct numerical simulation data, the topological and dynamical properties of vorticity tube segments are explored, such as the scale distribution and chirality. The characteristic parameters have strong influence on the enstrophy production and vorticity stretching. Consequently the common knowledge in turbulence dynamics that vorticity is more stretched than compressed need to be rectified in the vorticity tube segment context.
\end{abstract}

vorticity vector, vorticity tube segment, chirality, turbulent flow structure

PACS: $47.27 .-\mathrm{i}, 47.27 . \mathrm{Eq}, 47.27 . \mathrm{Nz}, 47.40 . \mathrm{Ki}, 47.85 . \mathrm{Gj}$

doi: 10.1360/SSPMA-2019-0086 